by means of their absorption spectra. For this purpose, pulsed light sources have been developed which give intense flashes of light of about $1 \mu \mathrm{sec}$. duration. These flashes will be synchronized with the pulse of radiation so as to obtain a series of absorption spectra at various intervals after the irradiation. In order to analyse the spectra, a highspeed rotating mirror with frictionless bearing has been built. In another series of experiments the effects of radiations on tissue enzymes are being studied, and experiments are in preparation for the investigation of the relationship between dose-rate and biological effects on the living cell by the use of the tissue-culture technique.

':Bareford, C. F., and Kelliher, M. G., Philips Tech. Rev., 15, 1 (1953).

\section{RELAXATION AND FLOW IN GLASSES}

$\mathrm{T}$

HE British Society of Rheology held a sym. posium at Queen Mary College (University of London) on January 28 on the subject of "Relaxation and Flow in Glasses". The theme was introduced by Prof. G. O. Jones (Queen Mary College), who gave an account of the nature and physical properties of 'glass'-using the term in its broadest connotation to cover such substances as glycerol, glucose, water, ordinary inorganic glasses and silica, having widely differing chemical behaviour. In this sense the term has essentially a thermodynamic significance, and it is constructive to approach the study of glasses from this point of view.

In the thermodynamic hierarchy, glass is a doubly unstable liquid, the progress of which towards stability is inhibited because of its high viscosity. It is, first, unstable relative to any ordered crystalline state and will, under certain conditions, crystallize or 'devitrify'. However, in normal use, this does not occur. Of greater physical significance in the study of glass is its instability relative to the state of internal equilibrium which corresponds to ordinary supercooling of a liquid. The tempersture at which the viscosity is $10^{18}$ poise (the 'transformation temperature', $T_{g}$ ) marks a rough boundary above which a glass-forming system is merely a supercooled liquid and below which it is a glass. At any temperature below $T_{g}$, the glass takes a finite time to reach the state of internal equilibrium corresponding to the particular temperature. The progressive approach to this stato-often described as 'stabilization'-is normally shown macroscopically as a contraction. The significance of the figure $10^{13}$ is only that for most substances it corresponds to a Maxwell relaxation time of the order of the duration of ordinary experimental observations. As the temperature is lowered below $T_{g}$, the microscopic adjustments necessary for the attainment of the configuration appropriate to the actual temperature take longer and longer times. This has great practical significance in the manufacture of optical glass.

The rheological properties of a glass are of special interest since they can be assumed to imitate-but $\mathrm{n}$ slow motion-those of ordinary liquids. The reactions to an applied shear stress consist of a reversible elastic contribution-partly instantaneous and partly delayed or 'relaxing' - and an irreversible portion which is Newtonian viscous flow. Under a uniform compressive stress, there is, of course, no viscous flow, but again the two reversible reactions appear. The almost exact formal correspondence between the delayed reaction in this case and the process of stabilization is of special interest.

By varying the temperature, the relative importance of the separate reactions to applied stress may be varied, and they can be studied individually by simple methods. At temperatures well above $T_{g}$, only the viscous flow is normally of importance. Near $T_{g}$ the three types of reaction are of about equal magnitude in the time-scale of ordinary observations, and this gives rise to the possibility of observing frozen-in stresses between adjacent layers when glass in which a temperature gradient exists is cooled through the neighbourhood of $T_{g}$. The removal of these stresses (often known as 'strain') is the main function of 'annealing' processes in commercial glassware.

At sufficiently low temperatures the instantaneous elastic strain preponderates over the other reactions to applied stress, and glass (though still thermodynamically an unstable liquid) becomes in its mechanical properties the model of a brittle solid.

Mr. R. W. Douglas (Research Laboratories, General Electric Co., Ltd., and professor-designate of glass technology in the University of Sheffield) spoke of his work in collaboration with Mr. J. O. Isard on the density of fused silica near $T_{g}$, where, of course, physical properties may be funotions of time as well as of other parameters. Because of its high transformation temperature (about $1,200^{\circ} \mathrm{C}$ ), silica has previously been somewhat inaccessible to such studies, and special methods have to be used. The typical experiment here consisted of heating the specimen of silica for a given interval at constant temperature, cooling quickly by withdrawing it from the furnace, and then measuring its density at room temperature. The specimen was again placed in the furnace and the whole process repeated until the measured density showed a constant value. A density thus measured contains, of course, a contribution from the thermal contraction during cooling to room temperature, and the coefficient of expansion governing this would be expected to change with time. In order to be able to make use of these measurements at room temperature to estimate the density at the original heating temperature, it was necessary to determine separately the effect of the heat treatment on the coefficient of expansion. The results show that this contribution accounts for about half the observed change in density.

The corrected equilibrium densities were found to increase as the temperature was increased in the range $1,050^{\circ}-1,300^{\circ} \mathrm{C}$., indicating that here fused silica has a negative coefficient of expansion if sufficient time is allowed for the atomic configuration to reach equilibrium. This behaviour appears to be analogous to that shown by water between $0^{\circ}$ and $4^{\circ} \mathrm{C}$., where the increase in density with rising temperature is attributed to the collapsing of the directed bonds. The well-known fact that the equilibrium configuration in glasses can be approached from both sides was demonstrated in these experiments for the case of silica.

Mr. Douglas went on to describe experiments on the release of mechanical stress in silica. It has long been known that the release of stress in glasses-for example, in annealing processes-does not follow the behaviour of the simple Maxwell body. Deviations from this behaviour must be partly due to the variation of viscosity with time and partly to the influence of delayed elastic (or visco-elastic) con- 
tributions. Since the rates of approach to equilibrium in silica near $T_{g}$ have been established from the experiments on density changes, an attempt wos made to estimate how far the deviations from Maxwellian behaviour are due to varying viscosity by making measurements of the release of stress from time to time during the slow approach of a specimen of silica towards its equilibrium configuration. The specimen consisted of a thick-walled cylinder in which a slot had been cut. The stress could be introduced by inserting a wedge of fused silica into this slot, and the magnitude of the stress determined by observing the birefringence. The rate of release of stress (at constant strain) was found to decrease when the specimen was heated. Eventually it reached a constant value after a time about equal to that which had been found necessary for the attainment of the equilibrium density at the same temperature. However, even at equilibrium, the release of stress did not follow the pattern expected from a Maxwell body, and an important contribution from viscoelasticity appeared to be present. The experiments were not regarded as decisive because it was found that as the material approached equilibrium it appeared to become more and more inhomogeneous. This behaviour was attributed to the fact that the viscosity after first cooling from a high temperature would have nearly the same value throughout the specimen, but as the configuration reached equilibrium, differences in viscosity due to physical inhomogeneities might become more marked.

Dr. R. O. Davies (Queen Mary College) spoke of the possibility of giving a thermodynamic treatment of the process of attaining configurational equilibrium (or stabilization).

In order to do this, one. must assume that the instantaneous state is fixed not merely by the pressure and temperature (say) but by one or more internal ordering parameters as well. The principle involved is not new, but has been used in chemical thermodynamics since the time of Gibbs. (In chemistry the internal parameters are 'degrees of reaction', describing the progress of chemical changes which can take place in the system.) With glasses the simplest procedure is that due to Tool, who introduced and used a 'fictive temperature' $(\bar{T})$, giving a measure of the internal state of the glass. When $\bar{T}$ is equal to the real temperature $(T)$, the glass is in thermodynamic equilibrium and has its equilibrium structure; when it is not, the structure is that of a glass which would be in equilibrium at a temperature higher or lower than $T$ (according as $\bar{T}$ is greater or less than $T$ ).

Alternatively, a 'fictive pressure' might be introduced. This is especially convenient in the discussion of experiments in which the pressure on the glass is varied, and can be used to give a qualitative explana. tion of some aspects of the recent experimental observations of P. W. Bridgman and I. Simon on volume changes in glass at very high pressure.

Tool's idea can be fitted into a rigorous thermodynamic formalism which-with appropriate extensions-should be applicable to any isotropic process in glasses. The main results for a single ordering parameter are: $(a)$ a set of thermodynamic relations connecting the properties of a glass with those of the corresponding equilibrium liquid, $(b)$ a means of predicting the kinetic behaviour under various experimental conditions (for example, isothermalisobaric, adiabatic-isobaric, etc.) in terms of that observed under any one set of conditions.
Experimentally it is found that in no case which can be checked are the predictions under $(a)$ exactly fulfilled. Those of $(b)$ have never been tested. The reason for the failure of $(a)$ is that one ordering parameter is not enough. This is especially conspicuous in the special case of borosilicate glass. To remedy this, more parameters must be introduced, and the limiting case is that of an infinity of parameters and a continuous distribution of relaxation times. Thus the thermodynamic theory becomes similar to the treatment of the general isotropic response of a linear body-a problem familiar to rheologists. The thermodynamic approach is, however, more flexible and attractive than the purely mechanical treatment.

It is clearly desirable to extend the thermodynamic treatment so as to deal with shearing motion as well. Here a curious situation appears. We should like to be able to exhibit delayed elasticity and flow as a special case of stabilization. It is possible to deal with delayed elasticity provided we exclude per. manent deformation and admit a tensorial degree of order instead of a scalar quantity. Alternatively, it is possible to derive the Stokes-Navier equations for the case where there is no delayed elasticity but only permanent deformation. But the central problem of combining both reversible and irreversible fluidity within a single thermodynamic framework seems to be more difficult.

\section{PROJECT 'SHOWER' : AN INVESTIGATION ON WARM RAIN IN THE HAWAIIAN ISLANDS}

\author{
BY P. SQUIRES \\ Division of Radiophysics, Commonwealth Scientific and \\ Industrial Research Organization, Sydney
}

$\mathrm{O}^{\mathrm{N}}$ $\mathrm{NE}$ of the most interesting problems in the subject of the physics of rain is posed by rain which falls from clouds wholly warmer than freezing, often called simply 'warm rain'. The classical theory of the formation of raindrops expounded in 1933 by Bergeron envisaged a cloud of supercooled water droplets in which, as cooling proceeded, ice crystals began to form, usually at temperatures around $-10^{\circ}$ to $-15^{\circ} \mathrm{C}$. However, over the past ten years or so, many cases of 'warm rain' have been reported, and, after some hesitation, its existence is now universally accepted.

The basic problem in the formation of this sort of rain is to explain how a single cloud process can sometimes give rise to two families of drops : on one hand, cloud drops, and on the other, raindrops which are of the order of $10^{5}$ times greater in mass. Some quite drastic form of selection in favour of a few of the droplets seems to be indicated. In the Bergeron process the singular character of the ice crystal in a cloud of supercooled water provides a key which seems adequate. In warm elouds it is not obvious what determines the selective growth of a small number of drops.

Condensation nuclei vary widely in size and effectiveness. This no doubt causes some cloud droplets, formed on the larger or more effective nuclei, to outgrow others. Nevertheless, it is generally agreed that this effect is quite inadequate to explain 